\title{
Effective Scattering Coefficient of the Cerebral Spinal Fluid in Adult Head Models for Diffuse Optical Imaging
}

\author{
Anna Custo ${ }^{1,2}$, William M. Wells III ${ }^{1,3}$, Alex H. Barnett ${ }^{2}$, Elizabeth M.C. Hillman ${ }^{2}$ and \\ David A. Boas ${ }^{2}$ \\ ${ }^{1}$ Computer Science and Artificial Intelligence Laboratory, Massachusetts Institute of \\ Technology, Cambridge, MA 02139 \\ ${ }^{2}$ Athinoula A. Martinos Center for Biomedical Imaging, Massachusetts General Hospital, \\ Harvard Medical School, Charlestown, MA 02129 \\ ${ }^{3}$ Department of Radiology, Brigham and Women's Hospital, Harvard Medical School, Boston, \\ MA 02115
}

\section{Abstract.}

Efficient computation of the time-dependent forward solution for photon transport in a head model is a key capability for performing accurate inversion for functional Diffuse Optical Imaging (DOI) of the brain. The diffusion approximation to photon transport is much faster to simulate than the physically-correct radiative transport equation (RTE), however, it is commonly assumed that scattering lengths must be much smaller than all system dimensions and all lengths for the approximation to be accurate. Neither of these conditions is satisfied in the cerebrospinal fluid (CSF). Since line-of-sight distances in the CSF are small, of the order a few $\mathrm{mm}$, we explore the idea that the CSF scattering coefficient may be modeled by any value from zero up to the order of the typical inverse line-of-sight distance, or about $0.3 \mathrm{~mm}^{-1}$, without significantly altering 
calculated detector signals or partial pathlengths relevant for functional measurements. We demonstrate this in detail using Monte Carlo simulation of the RTE in a three-dimensional head model based on clinical MRI data, with realistic optode geometries. Our findings lead us to expect that the diffusion approximation will be valid even in the presence of the CSF, with consequences for faster solution of the inverse problem.

OCIS codes: $170.6960,170.3660,170.5280,170.6920$

\section{Introduction}

Diffuse Optical Imaging (DOI) is a relatively new method used to image blood oxygenation in vivo. It uses near infrared light and has the advantage of low cost and portability. The success of diffuse optical imaging techniques is due to the properties of near infrared light in biological tissue. The absorption coefficient $\left(\mu_{\mathrm{a}}\right)$ depends on the total hemoglobin concentration and oxygenation within the tissue; therefore, calculating $\mu_{\mathrm{a}}$ provides useful information about the physiological conditions of the tissue ${ }^{1}$. For instance, during the last few years DOI has been tested for application to imaging breast cancer ${ }^{2-9}$ and brain function ${ }^{10-16}$.

In DOI near infrared light is scattered in a medium with optical properties $x$, and some fluence $y$ is recorded at the detectors' positions. Solving an imaging problem (described as $f(x)=y$; solved for $x$ ) requires a good combination of a forward and an inverse model. The forward problem models the process producing the set of measurements (establishing the rules to calculate $f(x)$ ). The inverse problem arises when it is necessary to recover an image of the optical properties of the medium from the observed data (i.e. $\left.x=f^{-1}(y)\right)$. DOI image reconstruction problem is ill posed; hence the inverse procedure typically involves use of 
regularization techniques ${ }^{17,18}$. Because of its relatively poor spatial resolution, DOI is increasingly combined with other imaging techniques, such as MRI and X-Ray, which provide high-resolution structural information to guide the characterization of the unique physiological information offered by DOI ${ }^{8,19-22}$.

In recent years, several papers have been published by Okada et al ${ }^{23-26}$, Arridge et al ${ }^{27-28}$, Firbank at al ${ }^{29}$, and Hielscher et al ${ }^{30}$ exploring a variety of models of light propagation in highly scattering media with properties similar to those found in the human head. The low scattering properties of the Cerebral Spinal Fluid (CSF), surrounding the space between the brain and the Dura mater ${ }^{31}$ has been of particular concern in the development of an accurate photon migration forward problem for the human head as the diffusion equation is known to provide inaccurate solutions under such circumstances ${ }^{23,30-31}$. Controversy has continued as to whether this low scattering region (often called the void region) affects sensitivity in the underlying brain tissue ${ }^{24,32-33}$. As a result, several papers have been published exploring implementation of the radiative transport equation ${ }^{30,34}$ or hybrid combinations of the radiosity equation and the diffusion equation ${ }^{25-26}$. A number of papers have shown that in some idealized geometries, the transport equation is necessary to accurately describe photon migration in the presence of a low scattering region $25,30,33,35-36$. Transport-based modeling approaches, such as Monte-Carlo simulations, typically require excessively long processing times. This can make them unsuitable for use as the forward model in an effective image reconstruction algorithm. It is therefore desirable to adopt a faster alternative forward model with comparable accuracy. A solution of the diffusion equation, for instance via Finite Difference (FD), offers greater computational speed at the cost of reduced modeling accuracy 
30, 37 . It has been suggested ${ }^{23,37}$ that the diffusion equation may be sufficiently accurate given the folding nature of the low scattering region between the brain and the skull and the fact that this space is filled with connective tissue and blood vessels ${ }^{38}$.

The irregularity in the thickness of the CSF layer is of particular interest as it limits the average straight-line distance that a photon would travel in the "void" region. Thus, even if the scattering coefficient $\mu_{s}^{\prime}$ in the "void" region were zero, an increase in $\mu_{s}^{\prime}$ up to the value of the inverse typical straight-line distance is expected to cause little change in photon transport. In Barnett at al. ${ }^{37}$, we hypothesized that, since the line of sight of light propagation through CSF is likely to be in average $\leq 3 \mathrm{~mm}$, approximating the CSF reduced scattering coefficient with a value not larger than its inverse (which is $\sim 0.3 \mathrm{~mm}^{-1}$ ) would not introduce a large error. Therefore, keeping the overall goal of rapid diffusion modeling in mind, we conjecture that we can approximate CSF scattering coefficient with values between 0.1 and $0.3 \mathrm{~mm}^{-1}$ without introducing an error larger than $20 \%$.

DOI can be achieved using Continuous Wave (CW) and Time Domain (TD) and Frequency Domain measurements. Time Domain measurements of functional brain activity have recently been shown to provide dramatically enhanced sensitivity to cortical activation ${ }^{39-42}$. In this paper we consider both $\mathrm{CW}$ and TD propagation of light through the human head. We expect that our conclusions about the suitability of a larger scattering coefficient to approximate CSF will extend to Frequency Domain measurements. 
We recently began exploring the use of 3D MRI of the human head as a prior in an iterative reconstruction process of the optical properties of the different structures in the human head ${ }^{37}$ as well as for improving quantitative functional imaging ${ }^{22,43}$. This work required creation of an MRI-based anatomically correct 3D model of the head and brain. In this paper we use Monte Carlo (MC) modeling, which implements the transport equation ${ }^{44-45}$, to accurately simulate light propagation through this MRI-based 3D model of the human head. We simulated both time-resolved and continuous wave measurements to validate our hypothesis of an effective CSF scattering coefficient by measuring the error introduced when using a CSF scattering coefficient larger than a near zero value (we use the reference CSF scattering coefficient of $0.001 \mathrm{~mm}^{-1}$ ). Given this new larger effective $\mu_{\mathrm{s}}^{\prime}$, the conditions for validity of the diffusion approximation may hold to a much greater degree than previously thought, allowing accurate diffusion solution.

We calculate the deviation from our reference measurements of the photon fluence detected on the surface of the head and of the sensitivity to the brain. The results presented in this paper support our hypothesis that non-scattering CSF region can be treated with a larger scattering coefficient $\left(0.3 \mathrm{~mm}^{-1}\right)$ with only $20 \%$ difference in the measurements. This result will also have relevance for the debate on the exact CSF scattering coefficient, since it demonstrates that as long as it is less than the inverse typical line-of-sight distances, its exact value is not relevant. Thus, it is in principle possible that the diffusion equation may provide sufficiently accurate modeling of photon migration through the human head, greatly reducing computational expense. 


\section{Methods}

A. Head model and probe placement

We use segmented MRI data to create the head geometry that we employ for this study. Within this adult head model we distinguish three tissue types (extra-cerebral, CSF, and cerebral, as described in Table 1). The whole volume is voxelized in a cube with 128 voxels per side $\left(128^{3}\right.$ voxel in total, $2 \times 2 \times 2 \mathrm{~mm}^{3}$ each). A coronal slice of the $3 \mathrm{D}$ head model is shown in Fig. 1 with a voxel size of $1 \mathrm{~mm}$. This voxel size was increased to $2 \mathrm{~mm}$ for the simulation, decreasing the simulation run time and the memory requirement. In data not shown we established that this increase in voxel size does not significantly affect the simulated data. The grey diamond and circles on the surface of the head show the placement of the source and detectors respectively.

The optical properties for each tissue type provided in Table 1 were taken from the literature ${ }^{46-}$ 48. The refractive index was the same for all tissues and assumed to be 1 and scattering anisotropy $\mathrm{g}=0.01$. We chose value of $\mu_{\mathrm{s}}^{\prime}$ for CSF between 0.001 and $1 \mathrm{~mm}^{-1}$ to test our hypothesis that the value of CSF scattering coefficient can be modeled as the inverse of the average CSF layer thickness ${ }^{37}$. In our model we calculate $\mu_{s, C S F}^{\prime}=1 /$ average-thickness ${ }_{C S F}$, which gives us a $\mu_{s}^{\prime}$ of $\sim 0.25 \mathrm{~mm}^{-1}$.

Table 1: Optical Properties of the adult head model

\begin{tabular}{lccc}
\hline Tissue Type & $\begin{array}{c}\text { Reduced transport } \\
\text { scattering coefficient }\end{array}$ & Absorption & Tissue thickness \\
& {$\left[\mathrm{mm}^{-1}\right]$} & {$\left[\mathrm{mm}^{-1}\right]$} & \\
\hline Scalp and Skull & 0.86 & 0.019 & $3-8$ (scalp) 7-8 (skull)
\end{tabular}


For the purpose of this study, we used a linear probe geometry, in which the sources and detectors are positioned along a line on the surface of the head, all placed in the same coronal plane. The probe features a single source and 25 detectors placed at $12,14,16,18 \ldots, 60 \mathrm{~mm}$ from the source position. The source is located at the top of the head (see Fig. 1). We chose this particular arrangement of optodes to analyze the effect of the CSF scattering coefficient on the measured photon fluence as a function of the separation between the source and the detector. Furthermore, the same probe has been used in similar studies by Okada et al ${ }^{23}$.

\section{B. Solution of the Radiative Transport Equation}

Monte Carlo is a simple method that offers a great deal of freedom in defining geometries and optical properties based on the radiative transport equation ${ }^{44-45}$. The method models photon trajectories through heterogeneous tissues, reproducing the randomness of each scattering event in a stochastic fashion (a random seed is employed). When a photon is detected, its partial optical pathlength for each of the tissue types through which it passed are recorded in a history file. Monte Carlo methods have the disadvantage of being computationally expensive to use while obtaining a good signal to noise ratio (SNR) in highly scattering thick tissues. We typically run $10^{9}$ photons to achieve an appropriate SNR for the results presented in this paper. We run 11 independent Monte Carlo simulations of $10^{8}$ photons for each $\mu_{s}^{\prime}$ configuration so that we can calculate the standard deviation across the independent runs. Each simulation took about 12 hours (see Boas et al. ${ }^{45}$ for more details). By recording the pathlength of each photon, our Monte Carlo data can be converted to CW or TD. 
C. Calculation of the total fluence in time domain and continuous wave

In order to consider the time-resolved problem, from the data recorded by the Monte Carlo simulation we calculate the Temporal Point Spread Function (TPSF) using eq. (2) from ${ }^{45}$

$$
\Phi_{j}\left(t_{i}\right)=\frac{1}{N_{j}\left(t_{i}\right) \Delta t} \sum_{l=1}^{N_{j}\left(t_{i}\right)} \prod_{m=1}^{N_{R}} \exp \left(-\mu_{a, m} L_{j, l, m}\right) .
$$

where $\Phi_{j}\left(t_{i}\right)$ is the measured photon fluence at detector $j, N_{j}\left(t_{i}\right)$ is the number of photons collected at detector $j$ in a time-gate of width $\Delta t$ centered at time $t_{i}, \exp \left(-\mu_{a, m} L_{j, l, m}\right)$ accounts for the effects of absorption in each region where $L_{j, l, m}$ is the pathlength of photon $l$ through region $\mathrm{m}$, and the photon migration time is related to the photon pathlength by the speed of light in the medium. $N_{R}$ is the number of regions through which the photons migrate. The continuous-wave fluence is calculated by averaging over the time index $i$.

\section{Calculation of Partial Optical Pathlength Factor (PPF)}

Tissue scattering causes the photons to travel a greater distance than the geometric distance between the source and detector. The partial pathlength of light through each of the tissue types is defined as ${ }^{24,38-39,49}$

$$
\mathrm{PPF}_{\mathrm{m}}=\partial \mathrm{OD} / \partial \mu_{a, m}
$$

where $\mathrm{OD}=-\ln \left(\Phi / \Phi_{o}\right)$ and where $\Phi / \Phi_{o}$ is given by eq. (1) and $\Phi_{o}$ is the incident number of photons. The partial pathlength is thus easily derived from eq. (1)

$$
\operatorname{PPF}_{j, m}=\frac{\sum_{l=1}^{N_{j}\left(t_{i}\right)} \prod_{m=1}^{N_{R}} L_{j, l, m} \exp \left(-\mu_{a, m} L_{j, l, m}\right)}{\sum_{l=1}^{N_{j}\left(t_{i}\right)} \prod_{m=1}^{N_{R}} \exp \left(-\mu_{a, m} L_{j, l, m}\right)} .
$$




\section{Results}

A. Fluence and partial pathlength for continuous wave

In Fig. 2 we show the total measured fluence versus source-detector separation for different $\mu_{s, C S F}^{\prime}$ (Fig. 2a) and the fractional difference relative to $\mu_{s, C S F}^{\prime}=0.001 \mathrm{~mm}^{-1}$ (Fig. 2b). The total fluence is normalized by the incident fluence, i.e. the total number of photons launched in the Monte Carlo simulation. We see that the detected fluence varies little when the $\mu_{s, C S F}^{\prime}<=0.1$ $\mathrm{mm}^{-1}$ but that a significant difference occurs at larger $\mu_{s, C S F}^{\prime}$ for separations greater than $25 \mathrm{~mm}$. In Fig. $2 b$ we see that at small separations $(12-22 \mathrm{~mm})$ the fractional difference for increasing $\mu_{s, C S F}^{\prime}$ is less than $2 \%$, becoming significant $(>20 \%)$ at larger separations $(>32 \mathrm{~mm})$ when $\mu_{s, C S F}^{\prime}$ is $1.0 \mathrm{~mm}^{-1}$. This is to be expected because smaller separations are predominantly sensitive to scalp-skull and therefore do not probe the CSF layer.

In Fig. 3a we show the continuous-wave partial pathlength for the scalp-skull region and the brain region versus the source detector separation for different scattering coefficients in the CSF region. The results show that the sensitivity of the measurement to absorption changes does not change as $\mu_{s}^{\prime}$ in the CSF space increases from 0.01 to $0.1 \mathrm{~mm}^{-1}$, but that a change is observed with $\mu_{\mathrm{s}}^{\prime}=1.0 \mathrm{~mm}^{-1}$. This is consistent with the hypothesis that the sensitivity will change as the scattering length becomes smaller than the typical line of sight distance through the CSF which is approximately $3 \mathrm{~mm}$ in our model. 
In Fig. 3b,c we plot the fractional difference in the partial pathlength factor for the scalp / skull region and the brain region relative to that when $\mu_{s, C S F}^{\prime}=0.001 \mathrm{~mm}^{-1}$. In Fig. $3 \mathrm{~b}$ we observe a difference greater than $3 \%$ at larger separations $\left(>30 \mathrm{~mm}\right.$ ) for a CSF model with $\mu_{\mathrm{s}}^{\prime}=1.0 \mathrm{~mm}^{-1}$, and a difference less than $1 \%$ for $\mu_{s, C S F}^{\prime}=0.1$ and $0.01 \mathrm{~mm}^{-1}$. Fig. $3 \mathrm{c}$ shows the same fractional difference observed in the brain. In the brain, even at small separations we observe a large difference only when $\mu_{s, C S F}^{\prime}=1.0 \mathrm{~mm}^{-1}$, increasing from $20 \%$ to $47 \%$ as the separation increases. For the smaller $\mu_{s, C S F}^{\prime}$ we observe differences less than $10 \%$ at all separations.

To investigate the variation in more detail, we plot in Fig. 4-5 the deviation in detected photon fluence at the surface of the scalp (Fig. 4) and the deviation in the partial pathlength in the scalpskull and brain (Fig. 5) versus $\mu_{s}^{\prime}$ of the CSF at source-detector separations of 20,30, and 40 $\mathrm{mm}$. These results show that a change greater than $20 \%$ is not observed until $\mu_{\mathrm{s}}^{\prime}>0.3 \mathrm{~mm}^{-1}$, except at $40 \mathrm{~mm}$ separation. We believe that the large discrepancy observed at $40 \mathrm{~mm}$ is due to the weak signal reaching far detectors. These results support our hypothesis that we can treat this void-like region with a larger scattering coefficient $\left(0.1<\mu_{s}^{\prime}<0.3 \mathrm{~mm}^{-1}\right)$ and obtain similar results (errors between 10 and $20 \%$ for $\mathrm{CW}$ measurements with source-detector spacing of $<40$ $\mathrm{mm})$.

We also note from Figs. $3 \mathrm{a}$ and 5 that the brain PPF, which corresponds to sensitivity of the $\mathrm{CW}$ measurement to absorption changes in the brain, is higher when CSF $\mu_{s}^{\prime}$ is low than when CSF $\mu_{s}^{\prime}$ matches that of the brain. 
B. Fluence and Partial pathlength in the Time Domain (TD)

We further explore this result for time resolved photon migration. In Fig. 6a we show how the partial pathlength varies with photon transit time in the medium. These results confirm what we have seen in continuous wave: the time resolved fluence is approximately the same when $\mu_{s, C S F}^{\prime}$ is less than $0.1 \mathrm{~mm}^{-1}$ but a significant difference is observed for a larger scattering coefficient. In Fig. $6 \mathrm{~b}$ we quantify this change relative to the fluence when $\mu_{s, C S F}^{\prime}=0.001 \mathrm{~mm}^{-1}$ and observe a greater than $50 \%$ deviation for $\mu_{s, C S F}^{\prime}=1.0 \mathrm{~mm}^{-1}$ and mostly less than $8 \%$ deviation when $\mu_{s, C S F}^{\prime}$ $=0.1$ and $0.01 \mathrm{~mm}^{-1}$.

In Fig. 7 we explore how the CSF scattering coefficient affects the partial pathlength in the superficial layers and the brain versus time delay. The absolute partial pathlength in Fig. 7a shows that no difference is observed for $\mu_{s, C S F}^{\prime}=0.1$ and $0.01 \mathrm{~mm}^{-1}$ but that a difference is observed when $\mu_{\mathrm{s}}$ ' is increased further to $1.0 \mathrm{~mm}^{-1}$. The deviation with increasing $\mu_{s, C S F}^{\prime}$ relative to a $\mu_{s, C S F}^{\prime}$ of $0.001 \mathrm{~mm}^{-1}$ is shown in fig. $7 \mathrm{~b}$ and $7 \mathrm{c}$ for the superficial and brain regions respectively. Little deviation is observed in the superficial scalp-skull region as the deviation is never larger than $15 \%$. A more significant difference is observed for the brain, where the partial pathlength is underestimated by $20 \%$ to $50 \%$ for $\mu_{s, C S F}^{\prime}=1.0 \mathrm{~mm}^{-1}$ whereas the error is less than $20 \%$ when $\mu_{s, C S F}^{\prime}<0.3 \mathrm{~mm}^{-1}$. In all cases, the error is greater at the earliest photon migration times when brain sensitivity is weakest. 
Fig. 8 and 9 show the deviation in detected fluence (Fig. 8) and partial pathlength factor (Fig. 9) versus $\mu_{\mathrm{s}, C S F}^{\prime}$ at 20,30 , and $40 \mathrm{~mm}$ at a time delay of $2 \mathrm{~ns}$, where we see that changes greater than $20 \%$ only occur when $\mu_{s}^{\prime}>0.3 \mathrm{~mm}^{-1}$. These results further support that the CSF layer can be accurately characterized by a scattering coefficient value between 0 and $\sim 0.3 \mathrm{~mm}^{-1}$ and still provide detected fluence and brain sensitivity within an acceptable approximation error (between 10 and $20 \%)$.

As with the $\mathrm{CW}$ results, we note that for time-domain measurements the Partial pathlength in the brain also increases when CSF reduced scattering coefficient is low (see Fig. 7a, 9). Also, importantly, sensitivity to absorption changes in the brain is significantly enhanced at time delays greater than $1.5 \mathrm{~ns}$ with respect to $\mathrm{CW}$ sensitivity measurements (compare Fig. 3a and 7a).

\section{Conclusions}

We performed extensive simulation studies to quantify the deviation in photon migration measurements and sensitivity to brain given a range of CSF scattering coefficient values. Through the analysis of total fluence and partial optical pathlength using Monte Carlo simulations in an accurate MRI-based 3D head geometry we found that the CSF scattering coefficient can increase up to the inverse of its typical thickness without significant variation from a near zero scattering coefficient. The results support our initial hypothesis that an effective CSF scattering coefficient of approximately $0.3 \mathrm{~mm}^{-1}$ can be used. Under these circumstances it may be possible to obtain accurate solutions of the forward problem with the Diffusion 
Approximation. The advantage of using the diffusion approximation is that we can utilize faster algorithms to simulate photon migration in the adult head.

Our results also suggest that low-scattering CSF increases DOI measurement sensitivity to brain, in contrast to previous studies that assumed a simplified smooth CSF layer ${ }^{32,42,50-51}$. CSF may change depth sensitivity profile, but this does not mean that signal from the cortex is decreased in the presence of low-scattering CSF. We hypothesize that the presence of the CSF layer has the effect of concentrating measurement sensitivity to the more superficial layers of the cortex, but that the overall sensitivity to cortical hemodynamics is not adversely affected.

In conclusion, our results indicate that:

(1) Using a diffusion model with a CSF reduced scattering coefficient of approximately 0.3 $\mathrm{mm}^{-1}$ leads to measurements with errors no larger than $20 \%$, for both time-domain and CW.

(2) The sensitivity of DOI measurements to cortical activity are not adversely affected by the presence of CSF in a realistic 3D head geometry

(3) Time-Domain measurements can further reduce the effect of the CSF layer by increasing sensitivity to deeper tissues, in agreement with previous findings of Steinbrink et al ${ }^{47}$, Montcel et al $^{41}$ and Okada et al ${ }^{42}$.

\section{Acknowledgements}

We thank Jon Stott for useful conversations and guidance in the early stages of this work. This work was supported by NIH P41-RR14075, R01-EB002482 and NSF IIS 9610249. 


\section{References}

1. V. Chernomordik, D. W. Hattery, D. Grosenick, H. Wabnitz, H. Rinneberg, K. T. Moesta, P. M. Schlag and A. Gandjbakhche, "Quantification of optical properties of a breast tumor using random walk theory", Journal of Biomedical Optics 7, 80-7 (2002).

2. N. Shah, A. E. Cerussi, D. Jakubowski, D. Hsiang, J. Butler and B. J. Tromberg, "The role of diffuse optical spectroscopy in the clinical management of breast cancer", Dis Markers 19, 95105 (2003).

3. R. Choe, A. Corlu, K. Lee, T. Durduran, S. D. Konecky, M. Grosicka-Koptyra, S. R. Arridge, B. J. Czerniecki, D. L. Fraker, A. DeMichele, B. Chance, M. A. Rosen and A. G. Yodh, "Diffuse optical tomography of breast cancer during neoadjuvant chemotherapy: a case study with comparison to MRI", Med Phys 32, 1128-39 (2005).

4. X. Intes, J. Ripoll, Y. Chen, S. Nioka, A. G. Yodh and B. Chance, "In vivo continuous-wave optical breast imaging enhanced with Indocyanine Green", Med Phys 30, 1039-47 (2003).

5. P. Taroni, G. Danesini, A. Torricelli, A. Pifferi, L. Spinelli and R. Cubeddu, "Clinical trial of time-resolved scanning optical mammography at 4 wavelengths between 683 and $975 \mathrm{~nm}$,, J Biomed Opt 9, 464-73 (2004).

6. D. Grosenick, H. Wabnitz, K. T. Moesta, J. Mucke, M. Moller, C. Stroszczynski, J. Stossel, B. Wassermann, P. M. Schlag and H. Rinneberg, "Concentration and oxygen saturation of 
haemoglobin of 50 breast tumours determined by time-domain optical mammography", Phys Med Biol 49, 1165-81 (2004).

7. S. Srinivasan, B. W. Pogue, S. Jiang, H. Dehghani, C. Kogel, S. Soho, J. J. Gibson, T. D. Tosteson, S. P. Poplack and K. D. Paulsen, "Interpreting hemoglobin and water concentration, oxygen saturation, and scattering measured in vivo by near-infrared breast tomography", Proc Natl Acad Sci U S A 100, 12349-54 (2003).

8. A. Li, E. L. Miller, M. E. Kilmer, T. J. Brukilacchio, T. Chaves, J. Stott, Q. Zhang, T. Wu, M. Chorlton, R. H. Moore, D. B. Kopans and D. A. Boas, "Tomographic optical breast imaging guided by three-dimensional mammography", Appl Opt 42, 5181-5190 (2003).

9. M. A. Franceschini, K. T. Moesta, S. Fantini, G. Gaida, E. Gratton, H. Jess, W. W. Mantulin, M. Seeber, P. M. Schlag and M. Kaschke, "Frequency-domain techniques enhance optical mammography: initial clinical results", Proc Natl Acad Sci U S A 94, 6468-73 (1997).

10. A. Villringer, J. Planck, C. Hock, L. Schleinkofer and U. Dirnagl, "Near infrared spectroscopy (NIRS): a new tool to study hemodynamic changes during activation of brain function in human adults", Neuroscience Letters 154, 101-104 (1993).

11. Y. Hoshi and M. Tamura, "Detection of dynamic changes in cerebral oxygenation coupled to neuronal function during mental work in man", Neuroscience Letters 150, 5-8 (1993). 
12. M. A. Franceschini, S. Fantini, J. H. Thompson, J. P. Culver and D. A. Boas, "Hemodynamic evoked response of the sensorimotor cortex measured non-invasively with near infrared optical imaging", Psychophysiology 40, 548-560 (2003).

13. H. Koizumi, T. Yamamoto, A. Maki, Y. Yamashita, H. Sato, H. Kawaguchi and N. Ichikawa, "Optical topography: practical problems and new applications", Appl Opt 42, 3054-3062 (2003).

14. M. Pena, A. Maki, D. Kovacic, G. Dehaene-Lambertz, H. Koizumi, F. Bouquet and J. Mehler, "Sounds and silence: an optical topography study of language recognition at birth", Proc Natl Acad Sci U S A 100, 11702-11705 (2003).

15. E. Gratton, V. Toronov, U. Wolf, M. Wolf and A. Webb, "Measurement of brain activity by near-infrared light", J Biomed Opt 10, 11008 (2005).

16. T. Wilcox, H. Bortfeld, R. Woods, E. Wruck and D. A. Boas, "Using near-infrared spectroscopy to assess neural activation during object processing in infants", J Biomed Opt 10, $11010(2005)$.

17. S. R. Arridge, "Optical tomography in medical imaging", Inverse Problems 15, 41-93 (1999).

18. M. Bertero and P. Boccacci, Introduction to Inverse Problems in Imaging (IOP Publishing, Bristol, 1998). 
19. R. L. Barbour, H. L. Graber, J. Chang, S. S. Barbour, P. C. Koo and R. Aronson, "MRIguided optical tomography:prospects and computation for a new imaging method", IEEE Computation Science and Engineering 2, 63-77 (1995).

20. B. W. Pogue and K. D. Paulsen, "High-resolution near-infrared tomographic imaging simulations of the rat cranium by use of a priori magnetic resonance imaging structural information", Optics Letters 23, 1716-1718 (1998).

21. V. Ntziachristos, A. G. Yodh, M. Schnall and B. Chance, "MRI-guided diffuse optical spectroscopy of malignant and benign breast lesions", Neoplasia 4, 347-354 (2002).

22. D. A. Boas and A. M. Dale, "Simulation study of magnetic resonance imaging-guided cortically constrained diffuse optical tomography of human brain function", Appl Opt 44, 195768 (2005).

23. E. Okada, M. Firbank, M. Schweiger, S. R. Arridge, M. Cope and D. T. Delpy, "Theoretical and experimental investigation of near-infrared light propagation in a model of the adult head", Applied Optics 36, 21-31 (1997).

24. Y. Fukui, Y. Ajichi and E. Okada, "Monte Carlo prediction of near-infrared light propagation in realistic adult and neonatal head models", Applied Optics 2002 Optical Society of America Biomedical Topical Meeting, 7-10 April 2002 42, 2881-7 (2003). 
25. T. Hayashi, Y. Kashio and E. Okada, "Hybrid Monte Carlo-diffusion method for light propagation in tissue with a low-scattering region", Applied Optics 2002 Optical Society of America Biomedical Topical Meeting, 7-10 April 2002 42, 2888-96 (2003).

26. T. Koyama, A. Iwasaki, Y. Ogoshi and E. Okada, "Practical and adequate approach to modeling light propagation in an adult head with low-scattering regions by use of diffusion theory", Appl Opt 44, 2094-103 (2005).

27. S. R. Arridge, M. Schweiger, M. Hiraoka and D. T. Delpy, "A finite element approach for modeling photon transport in tissue", Medical Physics 20, 299-309 (1993).

28. S. R. Arridge, H. Dehghani, M. Schweiger and E. Okada, "The finite element model for the propagation of light in scattering media: a direct method for domains with nonscattering regions", Med Phys 27, 252-64 (2000).

29. M. Firbank, S. R. Arridge, M. Schweiger and D. T. Delpy, "An investigation of light transport through scattering bodies with non-scattering regions", Physics in Medicine and Biology 41, 767-83 (1996).

30. A. H. Hielscher, R. E. Alcouffe and R. L. Barbour, "Comparison of finite-difference transport and diffusion calculations for photon migration in homogeneous and heterogeneous tissues", Physics in Medicine and Biology 43, 1285-1302 (1998). 
31. E. M. C. Hillman, Experimental and theoretical investigations of near infrared tomographic imaging methods and clinical applications. (University College London, London, 2002).

32. H. Dehghani, S. R. Arridge, M. Schweiger and D. T. Delpy, "Optical tomography in the presence of void regions", J Opt Soc Am A Opt Image Sci Vis 17, 1659-70 (2000).

33. E. Okada and D. T. Delpy, "Effect of discrete scatterers in CSF layer on optical path length in the brain", Proceedings of the SPIE - The International Society for Optical Engineering Photon Migration, Diffuse Spectroscopy, and Optical Coherence Tomography: Imaging and Functional Assessment, 6-8 July 2000 4160, 196-203 (2000).

34. G. Bal, K. Ren, "Generalized diffusion model in optical tomography with clear layers", JOSA A 20, 2355-2364 (2003).

35. J. D. Riley, S. R. Arridge, Y. Chrysanthou, H. Dehghani, E. M. C. Hillman and M. Schweiger, "The radiosity diffusion model in 3D", Proceedings of the SPIE - The International Society for Optical Engineering Photon Migration, Optical Coherence Tomography, and Microscopy, 18-21 June 2001 4431, 153-64 (2001).

36. H. Dehghani, D. T. Delpy and S. R. Arridge, "Photon migration in non-scattering tissue and the effects on image reconstruction", Phys Med Biol 44, 2897-906 (1999). 
37. A. H. Barnett, J. P. Culver, A. G. Sorensen, A. Dale and D. A. Boas, "Robust inference of baseline optical properties of the human head with 3D segmentation from magnetic resonance imaging", Applied Optics 42, 3095-3108 (2003).

38. E. Okada and D. T. Delpy, "Near-infrared light propagation in an adult head model. I. Modeling of low-level scattering in the cerebrospinal fluid layer", Applied Optics 2002 Optical Society of America Biomedical Topical Meeting, 7-10 April 2002 42, 2906-14 (2003).

39. K. Uludag, M. Kohl, J. Steinbrink, H. Obrig and A. Villringer, "Cross talk in the LambertBeer calculation for near-infrared wavelengths estimated by Monte Carlo simulations", Journal of Biomedical Optics 7, 51-9 (2002).

40. J. Selb, J.J. Stott, MA Franceschini, A. G. Sorenson and D. A. Boas, "Improved sensitivity to cerebral dynamics during brain activation with a time-gated optical system: analytical model and experimental validation", Journal Biomed Optics 10 (1), 011013 (2005).

41. B. Montcel, R. Chabrier and P. Poulet, "Detection of cortical activation with time-resolved diffuse optical methods", Appl Opt 44, 1942-7 (2005).

42. E. Okada and D. T. Delpy, "The effect of a non-scattering layer on time-resolved photon migration paths", Proc SPIE Int Soc Opt Eng 3566, $2-9$ (1999). 
43. D. A. Boas, A. M. Dale and M. A. Franceschini, "Diffuse optical imaging of brain activation: approaches to optimizing image sensitivity, resolution, and accuracy", Neuroimage 23 Suppl 1, S275-88 (2004).

44. L. Wang, S. L. Jacques and L. Zheng, "MCML-Monte Carlo modeling of light transport in multi-layered tissues", Computer Methods and Programs in Biomedicine 47, 131-46 (1995).

45. D. A. Boas, J. P. Culver, J. J. Stott and A. K. Dunn, "Three dimensional Monte Carlo code for photon migration through complex heterogeneous media including the adult human head", Optics Express 10, 159-170 (2002).

46. D. A. Boas, G. Strangman, J. P. Culver, R. D. Hoge, G. Jasdzewski, R. A. Poldrack, B. R. Rosen and J. B. Mandeville, "Can the cerebral metabolic rate of oxygen be estimated with nearinfrared spectroscopy?" Physics in Medicine and Biology 48, 2405-18 (2003).

47. J. Steinbrink, H. Wabnitz, H. Obring, A. Villringer and H. Rinneberg, " Determining changes in NIR absorption using a layered model of the human head ", Phys. Med. Biol. 46, 879-896 (2001).

48. G. Strangman, M. A. Franceschini and D. A. Boas, "Factors affecting the accuracy of nearinfrared spectroscopy concentration calculations for focal changes in oxygenation parameters", Neuroimage 18, 865-879 (2003). 
49. E. Okada, M. Schweiger, S. R. Arridge, M. Firbank and D. T. Delpy, "Experimental validation of Monte Carlo and finite-element methods for the estimation of the optical path length in inhomogeneous tissue", Applied Optics 35, 3362-71 (1996).

50. H. Dehghani and D. T. Delpy, "Near-infrared spectroscopy of the adult head: Effect of scattering and absorbing obstructions in the cerebrospinal fluid layer on light distribution in the tissue", Applied Optics 39 (25), 4721-4729 (2000).

51. S. Takahshi and Y. Yamada, "Simulation of 3D light propagation in a layered head model including a clear CSF layer", OSA TOPS: Advances in Optical Imaging and Photon Migration 21, 2-6 (1998). 


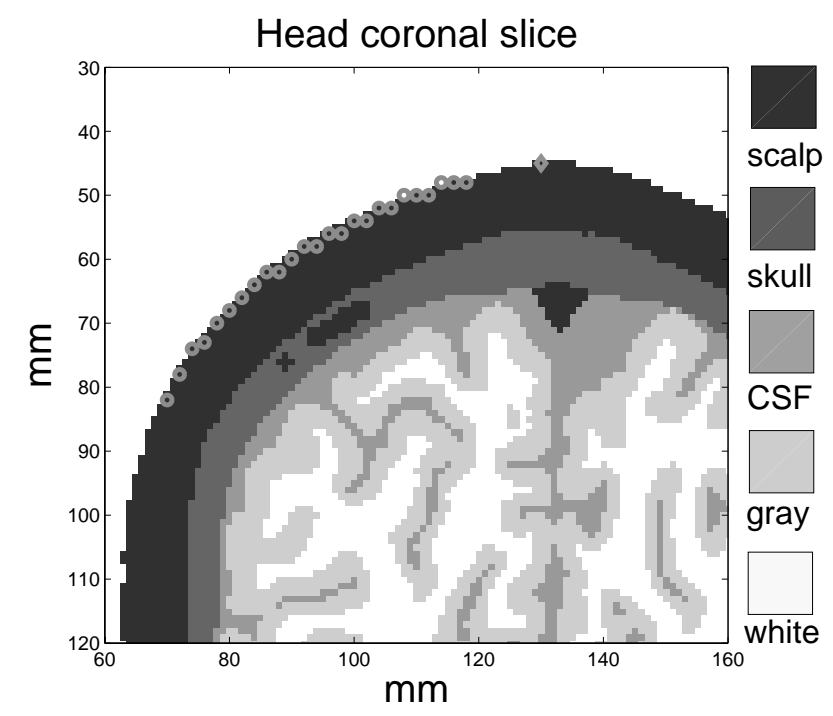

Fig. 1: Head geometry and probe placement. The grey diamond on the top of the head indicates the position of the single source and the grey circles show the position of the twenty five detectors. 


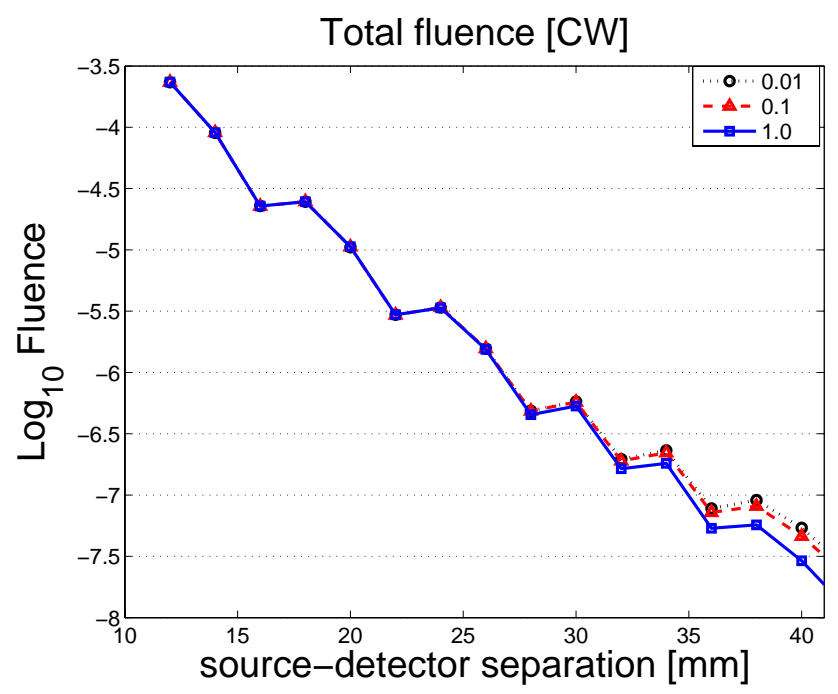

Fig. 2: (a) Total detected fluence simulated with Monte Carlo in continuous wave.

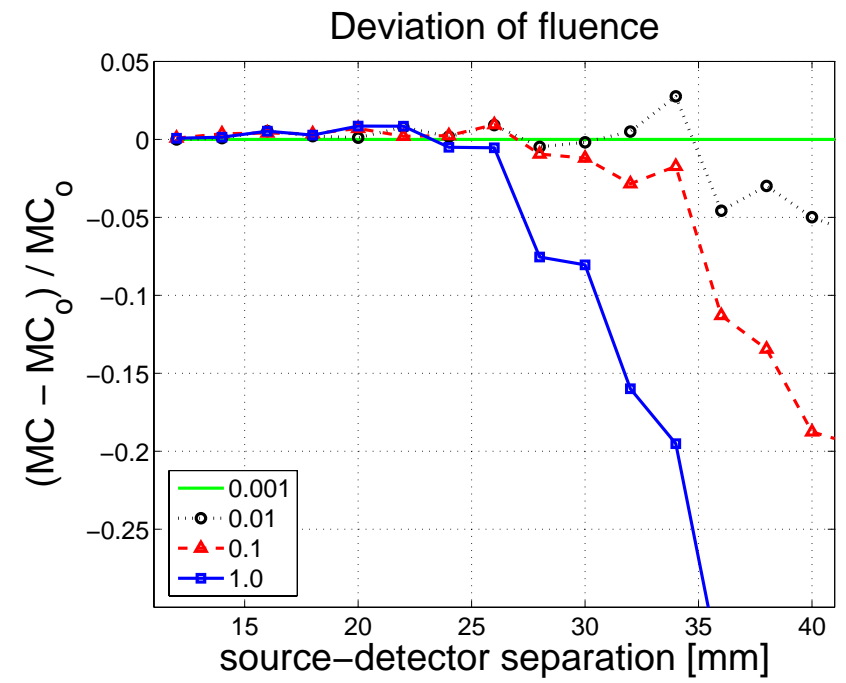

Fig. 2: (b) Relative fluence in continuous wave calculated with respect to $\mathrm{MC}_{\mathrm{o}}$, which is the Monte Carlo prediction when $\mu_{\mathrm{s}, \mathrm{CSF}}^{\prime}=0.001 \mathrm{~mm}^{-1}$. 

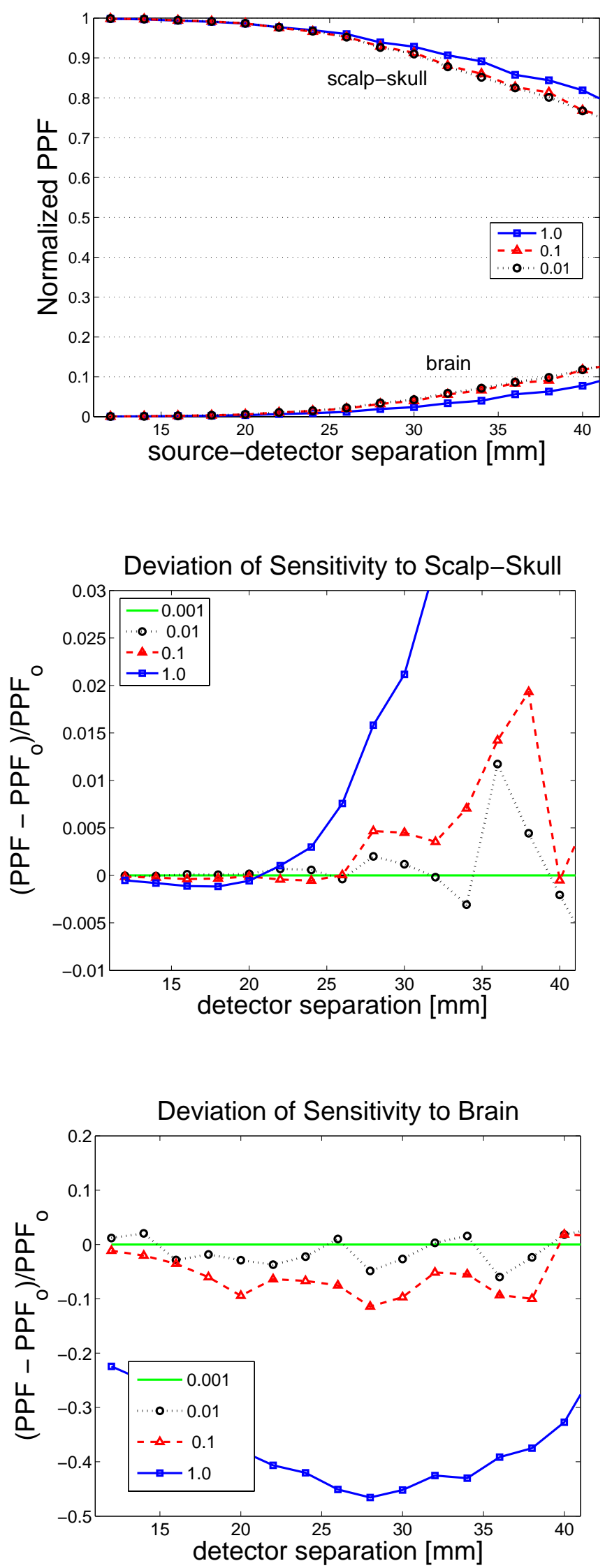

Fig. 3a: Monte Carlo normalized pathlength factor calculated versus separation for three different $\mu^{\prime}{ }_{\mathrm{s}, \mathrm{CSF}}: 0.01$, 0.1 and $1.0 \mathrm{~mm}^{-1}$ in continuous wave. The PPF is normalized by the total sensitivity to all tissue types.
Fig. 3b: Monte Carlo measure of relative sensitivity to scalp-skull layer versus separation when varying $\mu_{\mathrm{s}, \mathrm{CSF}}$ in continuous wave $\left(\mu^{\prime}{ }_{\mathrm{s}}=0.01,0.1\right.$ and 1.0 $\left.\mathrm{mm}^{-1}\right)$. The error is calculated with respect to $\mathrm{PPF}_{\mathrm{o}}$, which is the Monte Carlo prediction of PPF when $\mu_{\mathrm{s}, \mathrm{CSF}}^{\prime}=0.001$ $\mathrm{mm}^{-1}$.
Fig. 3c: Monte Carlo measure of relative sensitivity to brain versus separation when varying $\mu^{\prime}{ }_{\mathrm{s}, \mathrm{CSF}}$ in continuous wave $\left(\mu^{\prime}{ }_{s}=0.01,0.1\right.$ and $\left.1.0 \mathrm{~mm}^{-1}\right)$. The error is calculated with respect to $\mathrm{PPF}_{\mathrm{o}}$, which is the Monte Carlo prediction of PPF when $\mu_{\mathrm{s}, \mathrm{CSF}}^{\prime}=0.001 \mathrm{~mm}^{-1}$. 

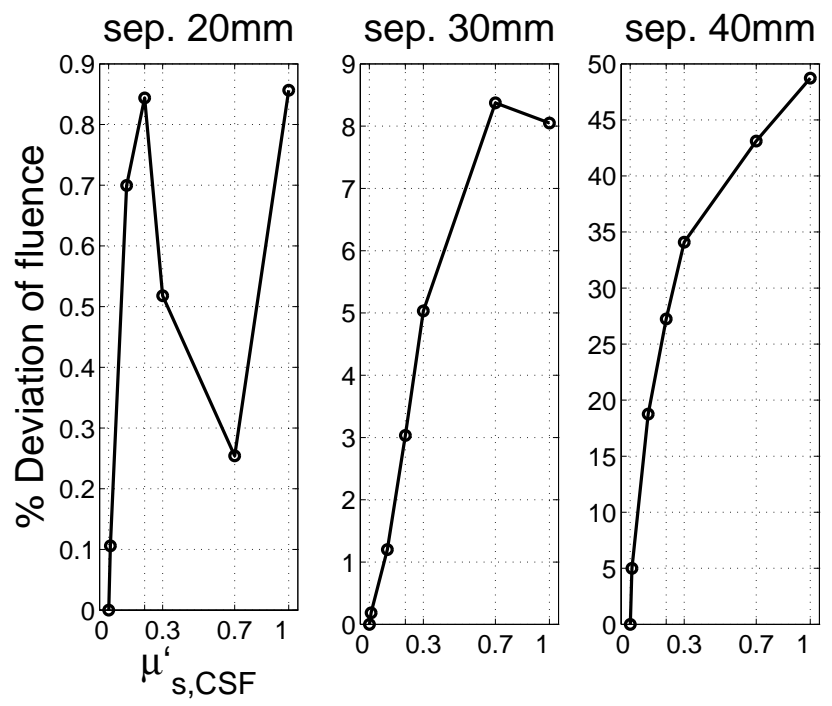

Fig. 4: Deviation of fluence as a function of $\mu_{\mathrm{s}, \mathrm{CSF}}^{\prime}$ (percentage changes with respect to $\mu_{\mathrm{s}, \mathrm{CSF}}^{\prime}=0.001 \mathrm{~mm}^{-1}$ ). CSF scattering coefficient varies from 0.001 to $1.0 \mathrm{~mm}^{-1}$. The data are calculated via Monte Carlo simulations in continuous wave using $\mu_{\mathrm{s}, \mathrm{CSF}}$ values of $0.001,0.01,0.1,0.2,0.3,0.7$, and 1.0 . 

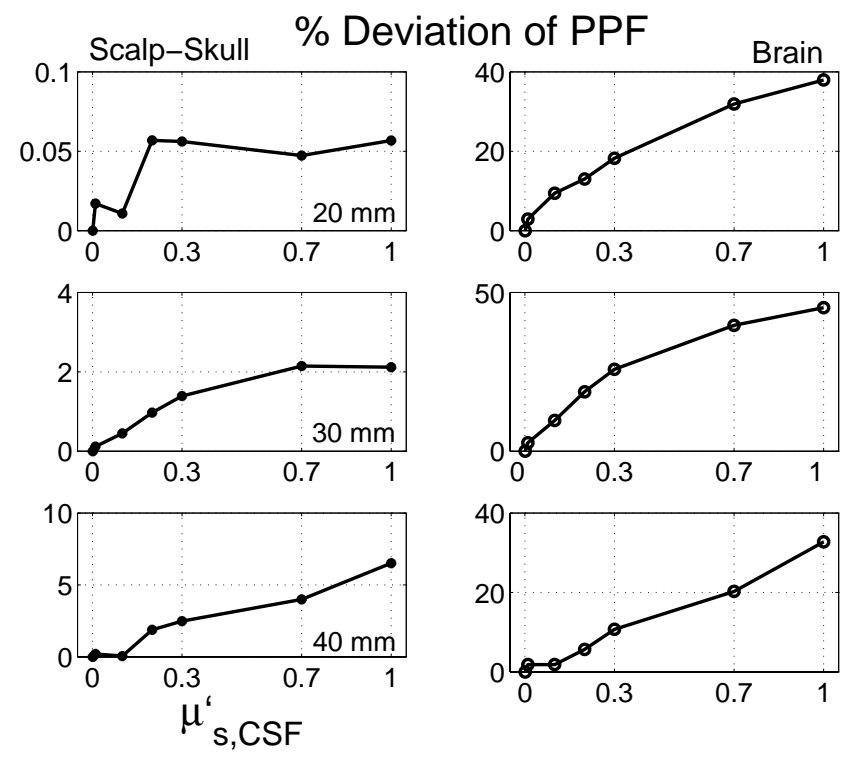

Fig. 5: Percent deviation of Partial pathlength as a function of $\mu^{\prime}{ }_{\mathrm{s}, \mathrm{CSF}}$. The deviation is calculated with respect to PPF measured when CSF $\mu_{\mathrm{s}, \mathrm{CSF}}^{\prime}=0.001 \mathrm{~mm}^{-1}$. CSF scattering coefficient varies from 0.001 to $1.0 \mathrm{~mm}^{-1}$ taking values of $0.001,0.01,0.1$, $0.2,0.3,0.7$, and 1.0, and the pathlength factor is simulated with Monte Carlo in continuous wave. Results are shown for separations of 20, 30, and $40 \mathrm{~mm}$. 

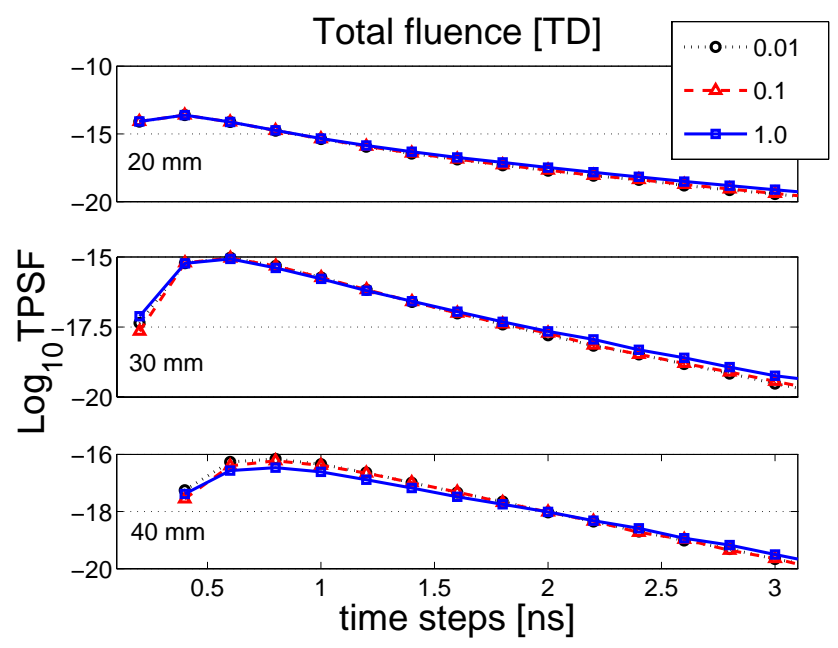

Fig 6: (a) Temporal Point Spread Function predicted by Monte Carlo.

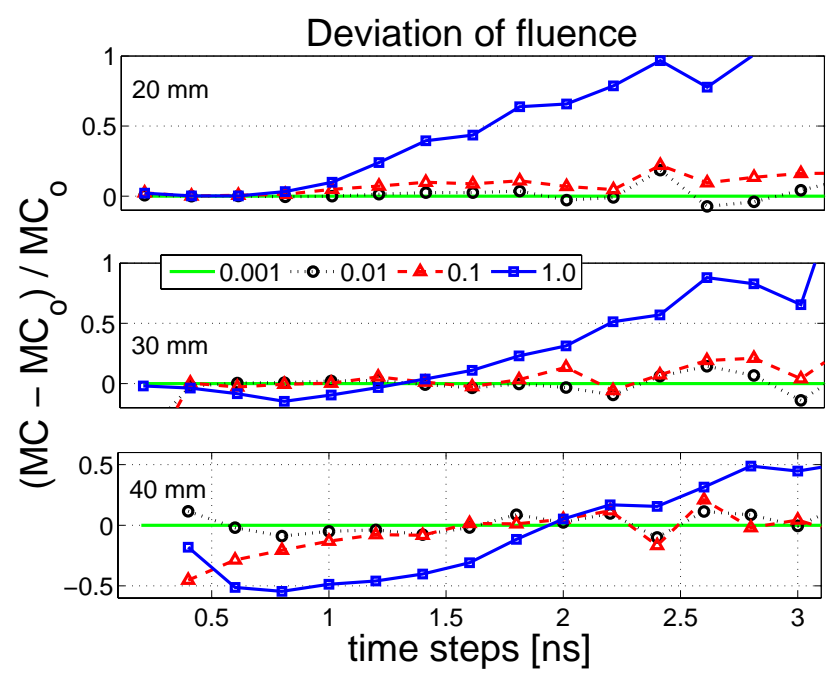

Fig 6: (b) Temporal Point Spread Function relative error with respect to the reference measurement $\mathrm{MC}_{\mathrm{o}}$, simulated with $\mu_{\mathrm{s}, \mathrm{CSF}}^{\prime}=0.001 \mathrm{~mm}^{-1}$. Results are shown for separations of 20,30 , and $40 \mathrm{~mm}$. 


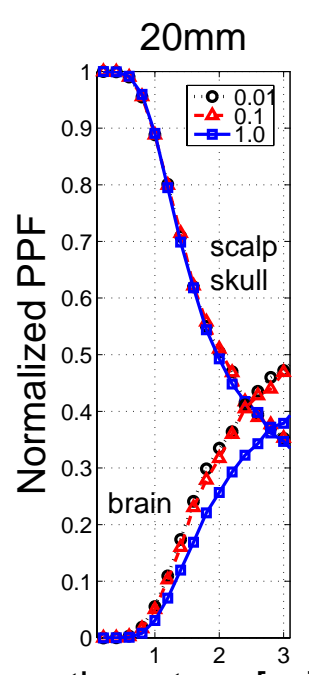

time steps [ns]
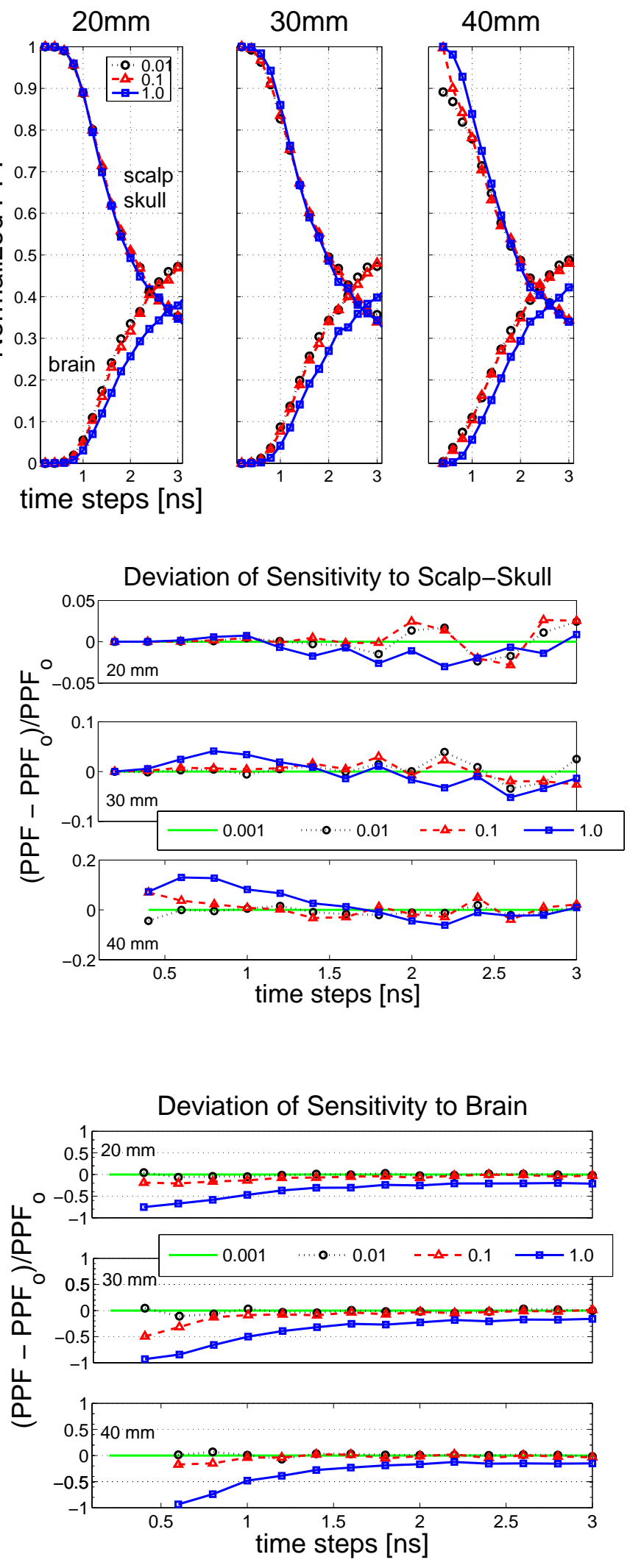

Fig. 7a: Monte Carlo prediction of the optical pathlength factor at three $\mu_{\mathrm{s}, \mathrm{CSF}}{ }^{\prime}: 0.01,0.1$ and $1.0 \mathrm{~mm}^{-1}$ versus time delay normalized by the total PPF. Results are shown for separations of 20,30 , and $40 \mathrm{~mm}$.

Fig. 7b: Relative sensitivity to absorption changes in the scalpskull layer when $\mu^{\prime}{ }_{\text {s,CSF }}=0.01,0.1$ and $1.0 \mathrm{~mm}^{-1}$ versus time delay as predicted by Monte Carlo. The reference measure of sensitivity to scalp-skull is given by simulating PPF when $\mu^{\prime}$ s,CSF is $0.001 \mathrm{~mm}^{-1}$.

Fig. 7c: Time resolved Monte Carlo predictions of the relative sensitivity to absorption changes in the brain when $\mu_{\mathrm{s}, \mathrm{CSF}}^{\prime}$ assumes the values $0.01,0.1$ and $1.0 \mathrm{~mm}^{-1}$. The reference measure of sensitivity to brain is given by simulating PPF when $\mu_{\mathrm{s}, \mathrm{CSF}}^{\prime}$ is $0.001 \mathrm{~mm}^{-1}$. Results are shown for separations of 20,30 , and $40 \mathrm{~mm}$. 

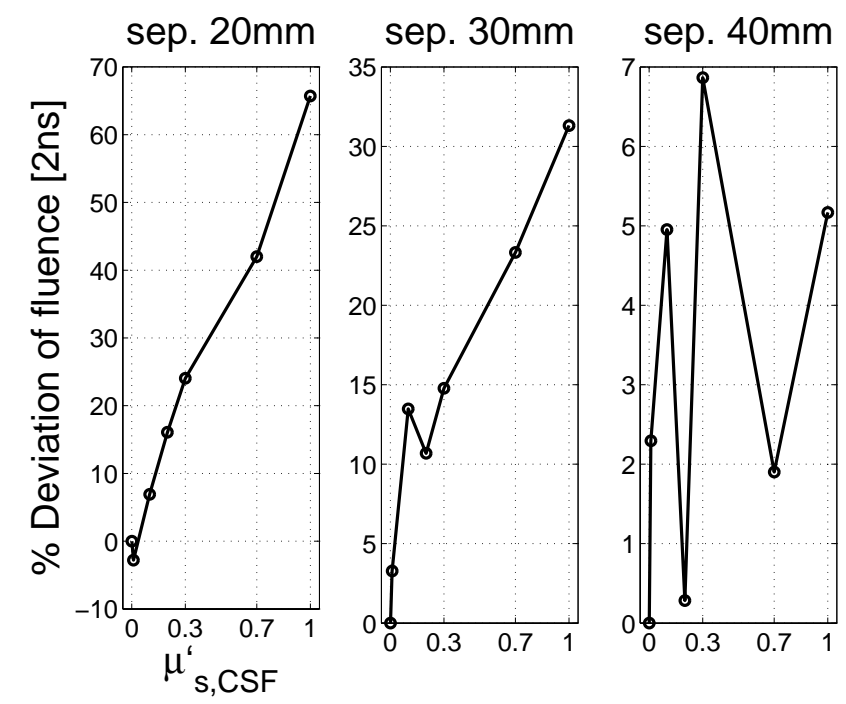

Fig. 8: Deviation of fluence as a function of $\mu^{\prime}{ }_{\mathrm{s}, \mathrm{CSF}}$ (percentage changes with respect to $\mu_{\mathrm{s}, \mathrm{CSF}}^{\prime}=0.001 \mathrm{~mm}^{-1}$ ) at 20,30 and $40 \mathrm{~mm}$ separation from the source at a time delay of $2 \mathrm{~ns}$. CSF scattering coefficient varies between 0.001 and $1.0 \mathrm{~mm}^{-1}$. 

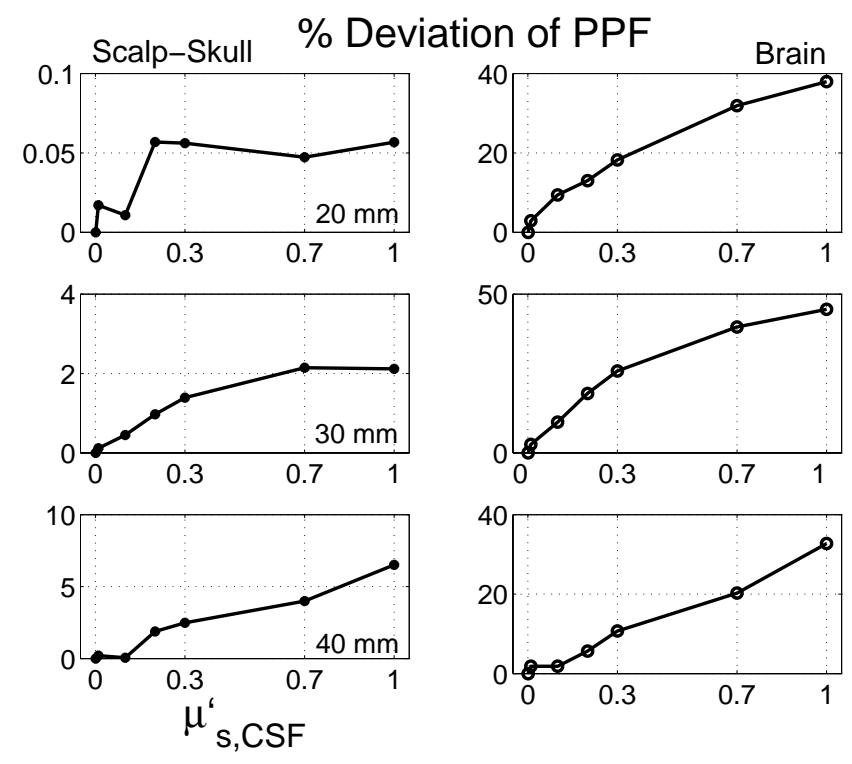

Fig. 9: Percent deviation of Partial pathlength as a function of $\mu_{\mathrm{s}, \mathrm{CSF}}^{\prime}$. The deviation is calculated with respect to PPF measured when CSF $\mu_{\mathrm{s}, \mathrm{CSF}}=$ $0.001 \mathrm{~mm}^{-1}$. The results are shown for source-detector separations of 20,30 and $40 \mathrm{~mm}$, for a time delay of $2 \mathrm{~ns}$. 


\section{Figure Captions}

Fig. 1: Head geometry and probe placement. The grey diamond on the top of the head indicates the position of the single source and the grey circles show the position of the twenty five detectors.

Fig. 2: (a) Total detected fluence simulated with Monte Carlo in continuous wave.

(b) Relative fluence in continuous wave calculated with respect to $\mathrm{MC}_{\mathrm{o}}$, which is the Monte Carlo prediction when $\mu_{s, C S F}^{\prime}=0.001 \mathrm{~mm}^{-1}$.

Fig. 3a: Monte Carlo normalized pathlength factor calculated versus separation for three different $\mu_{s, C S F}^{\prime}: 0.01,0.1$ and $1.0 \mathrm{~mm}^{-1}$ in continuous wave. The PPF is normalized by the total sensitivity to all tissue types.

Fig. 3b-c: Monte Carlo measure of relative sensitivity to scalp-skull layer (b) and brain (c) versus separation when varying $\mu_{s, C S F}^{\prime}$ in continuous wave $\left(\mu_{\mathrm{s}}{ }^{\prime}=0.01,0.1\right.$ and $\left.1.0 \mathrm{~mm}^{-1}\right)$. The error is calculated with respect to $\mathrm{PPF}_{\mathrm{o}}$, which is the Monte Carlo prediction of PPF when $\mu_{s, C S F}^{\prime}=0.001$ $\mathrm{mm}^{-1}$.

Fig. 4: Fluence changes as a function of $\mu_{s, C S F}^{\prime}$. CSF scattering coefficient varies from 0.001 to $1.0 \mathrm{~mm}^{-1}$. The data are calculated via Monte Carlo simulations in continuous wave using $\mu_{s, C S F}^{\prime}$ values of $0.001,0.01,0.1,0.2,0.3,0.7$, and 1.0 . 
Fig. 5: Partial pathlength absolute changes as a function of $\mu_{s, C S F}^{\prime}$. CSF scattering coefficient varies from 0.001 to $1.0 \mathrm{~mm}^{-1}$ taking values of $0.001,0.01,0.1,0.2,0.3,0.7$, and 1.0 , and the pathlength factor is simulated with Monte Carlo in continuous wave. Results are shown for separations of 20,30 , and $40 \mathrm{~mm}$.

Fig 6: (a) Temporal Point Spread Function predicted by Monte Carlo and (b) its relative error with respect to the reference measurement $\mathrm{MC}_{0}$, simulated with $\mu_{s, C S F}^{\prime}=0.001 \mathrm{~mm}^{-1}$. Results are shown for separations of 20,30 , and $40 \mathrm{~mm}$.

Fig. 7a: Monte Carlo prediction of the optical pathlength factor at three $\mu_{s, C S F}^{\prime}: 0.01,0.1$ and 1.0 $\mathrm{mm}^{-1}$ versus time delay normalized by the total PPF. Results are shown for separations of 20, 30, and $40 \mathrm{~mm}$.

Fig. 7b-c: (b) Relative sensitivity to absorption changes in the scalp-skull layer when $\mu_{s, C S F}^{\prime}=$ $0.01,0.1$ and $1.0 \mathrm{~mm}^{-1}$ versus time delay as predicted by Monte Carlo. The reference measure of sensitivity to scalp-skull is given by simulating PPF when $\mu_{s, C S F}^{\prime}$ is $0.001 \mathrm{~mm}^{-1}$ (c) Time resolved Monte Carlo predictions of the relative sensitivity to absorption changes in the brain when $\mu_{s, C S F}^{\prime}$ assumes the values $0.01,0.1$ and $1.0 \mathrm{~mm}^{-1}$. The reference measure of sensitivity to brain is given by simulating PPF when $\mu_{s, C S F}^{\prime}$ is $0.001 \mathrm{~mm}^{-1}$. Results are shown for separations of 20, 30, and $40 \mathrm{~mm}$. 
Fig. 8: Fluence changes as a function of $\mu_{s, C S F}^{\prime}$ for different separations at a time delay of $2 \mathrm{~ns}$. CSF scattering coefficient varies between 0.001 and $1.0 \mathrm{~mm}^{-1}$.

Fig. 9: Absolute changes in the partial optical pathlength versus $\mu_{s, C S F}^{\prime}$ at source-detector separations of 20, 30 and $40 \mathrm{~mm}$ for a time delay of $2 \mathrm{~ns}$. 